\title{
Design Insulated Hybrid Transformer of Application for AC Adapters of Smart devices
}

\author{
HeungGyoon(Harry) Choi ${ }^{1}$, KyuSun Chung ${ }^{1}, \mathrm{Ge} \mathrm{Li}^{2}$, XinLan $\mathrm{Li}^{2}$ \\ Department of Electrical Engineering, Hanyang University, Haengdang, Seongdong, Seoul, Korea \\ Circuit Drives Solution Div.,Samsung Electro-Mechanics Co.Meatan, Yeongtong, Suwon, Korea ${ }^{2}$
}

\begin{abstract}
Increasing strong demand for smaller insulated transformers, an ultra-small transformer has been developed by adopting an insulated hybrid structure with the size of W16.5 x L19.5 x H10.5 mm^3, whose volume is $65 \%$ smaller than conventional insulated transformers. Our new transformer has been improved in terms of noise reduction by $5-15 \mathrm{~dB}$, power efficiency increase by $2 \%$, and production efficiency by $50 \%$. Relevant analyses are to be given.
\end{abstract}

Keywords: AC Adapter, Insulated transformer, Hybrid structure, Small size.

\section{INTRODUCTION}

With continuing worldwide popularity, smart devices such as phones, pads, watches, and glassed has become more sophisticated. Consequently, their batteries consume more electricity and the lifespan of these batteries have also become shorter. For the conveniences of users of smart device users carrying AC adapters, smaller and lighter powers are in strong demand, which highlights the importance of smaller charging $\mathrm{AC}$ adapters. To meet the strong demand of smaller and lighter AC adaptor, we have developed a new transformer for AC adaptors of smart devices by utilizing insulated hybrid structure.

Figure 1 shows a conventional 10W AC adapter board and its parts. The transformer is the thickest part, accounting for approximately $30 \%$ of the AC adapter board. Thus, the transformer is one major item in making these $\mathrm{AC}$ adapters slimmer. Present power of AC adapters for smart-phones and tablet PCs is in the range of $5 \mathrm{~W}$ to $15 \mathrm{~W}$.

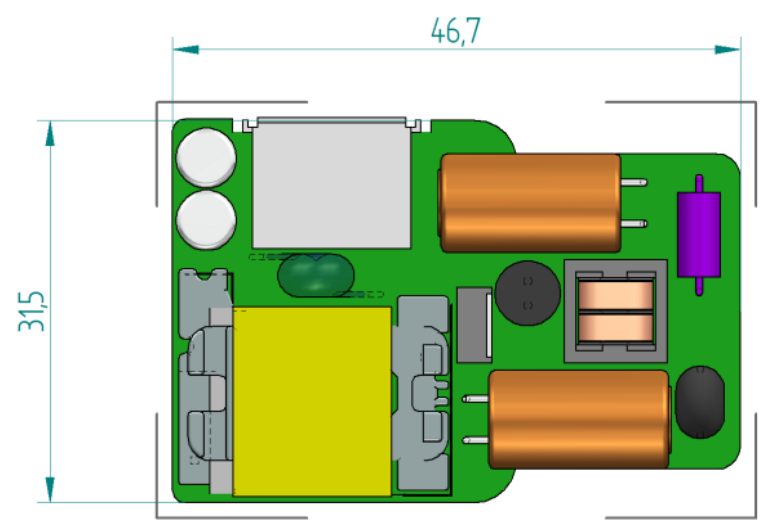

(a) Top view.

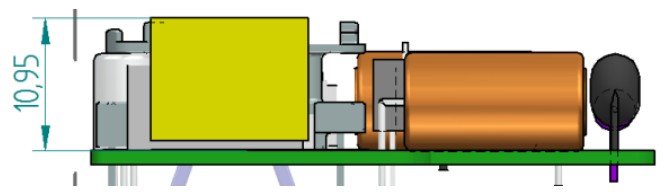

(b) Side view.

Fig.1. Conventional-type 10W AC Adapter.
We introduce an ultra-small transformer for $10 \mathrm{~W}$ AC adapters which has an insulated hybrid structure. We have evaluated our new transformer in terms of volume, noise level, power and manufacturing efficiencies. We have compared features of the conventional transformer with those of the new one, which showed that hybrid-type wires without bobbin have higher circuit efficiency than the conventional one and its transformer is approximately $45 \%$ smaller. Our new transformer also meets the characteristics of EMI and allowed for a shorter manufacturing process of transformer, increasing the mass production efficiency by more than $50 \%$ than the conventional one.

\section{CIRCUIT AND TRANSFORMER STRUCTURE}

Figure 2 shows a block diagram of the AC adapter power supply. It is separated by an isolation power transformer.

Specifications for AC adapter power supply:

Input voltage : AC $85 \sim 265 \mathrm{~V}$

Output voltage : $5 \mathrm{Vdc}$

Output current : 0 2.0A

EMI specifications:

CISPR22,FCC_PART15,EN550022

Safety specifications

EN60950/UL60950 AND IEC60950

Flyback Converter

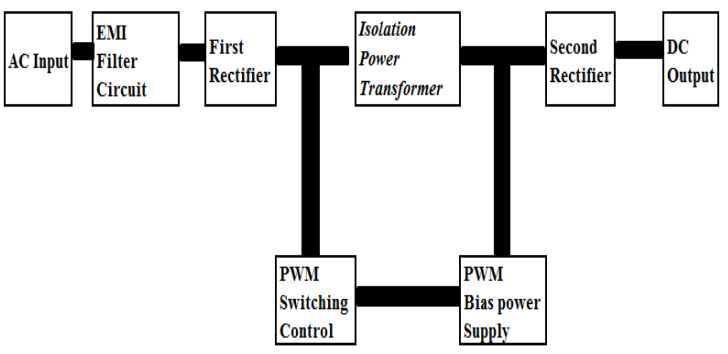

Fig.2. Block diagram of the AC adapter power supply. (PWM: Pulse Width Modulation). 


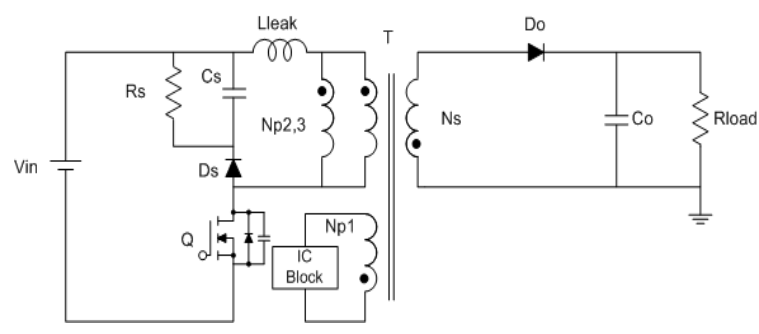

Fig.3. Flyback converter

Figure 3 represents a Flyback converter with the leakage inductance concentrated in primary winding. In the moment in which the MOSFET(Q) in turned off, current in the primary winding will try to reach zero, but the leakage inductance makes that the current does not change so quickly. An over-voltage will appear in the leakage inductance. This operation model and its current are determined based on leakage inductance as shown below equation $^{[7-10]}$.

$$
\begin{aligned}
& I_{p}(s)=\frac{\left(V_{g}+n V_{o}-v_{d s}\left(t_{3}\right)\right) c_{o s s}+i_{p}\left(t_{3}\right) L_{k} C_{o s s} s}{L_{k} C_{o s s} S^{2}+1}
\end{aligned}
$$

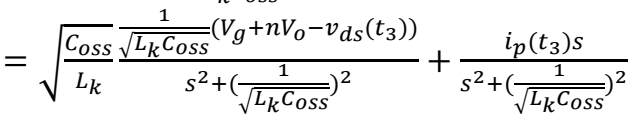

$$
\begin{aligned}
& i_{p}(t)=\left(V_{g}+n V_{o}-v_{d s}\left(t_{3}\right)\right) \sqrt{\frac{C_{\text {oss }}}{L_{k}}} \sin \left(\frac{1}{\sqrt{L_{k} C_{\text {oss }}}} t\right)+ \\
& i_{p}\left(t_{3}\right) \cos \left(\frac{1}{\sqrt{L_{k} C_{\text {OSS }}}} t\right)
\end{aligned}
$$

Figure 4 shows the drawing of transformer which has an insulated hybrid structure. This transformer does not require any bobbin unlike the conventional one. $\mathrm{Np} 1$ and $\mathrm{Np} 2$ of primary wires are made on multi-layered board and Ns1 made out of TIW is located on this board, which can be assembled into a transformer core. At this point, PCB needs to be shielded and the EMI level should go down in order to prevent EMI characteristics from being undermined. Since our new transformer does not require any bobbin, it can not only reduce the manufacturing processes by more than $50 \%$, but also the volume of transformer.

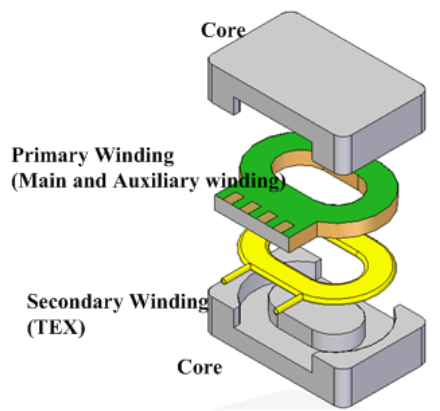

(a) Wire structure of transformer.

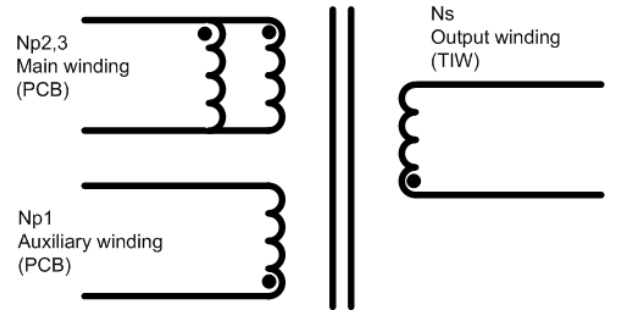

(b) Equivalent circuit of transformer.

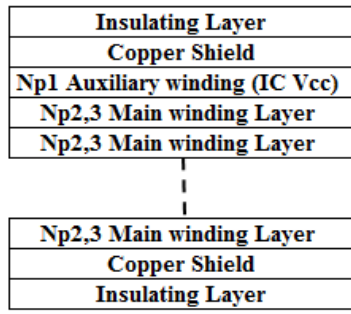

(c) Circuit structure of primary wires (Np1 and $\mathrm{Np} 2,3)$ Fig.4. The transformer structure with insulated hybrid structure.

\section{ANALYSIS OF THE TRANSFORMER WITH HYBRID INSULATED STRUCTURE}

A. Leakage Inductance

As AC adapters have a flyback converter-type circuit system, transformer inductance has a big impact on voltage surge and efficiency. The conventional one has small leakage inductance as primary and secondary wires have the interleaved structure (sandwich type) ${ }^{[1-5]}$.

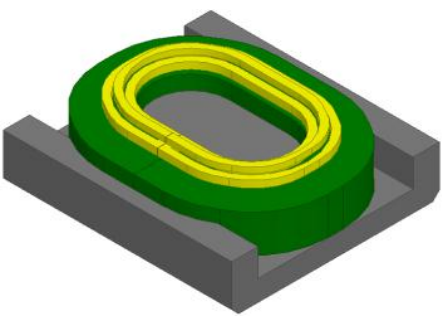

(a) 3D view of new type.

Secondary Winding (TEX)

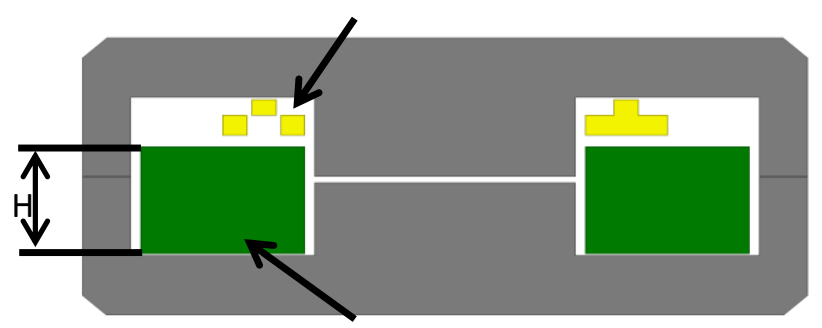

Primary Winding (PCB)

(b) 2D side view of the new type.

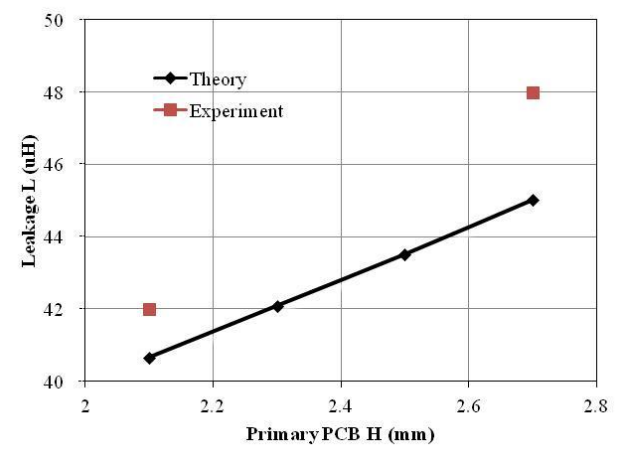

(c) The connection between primary PCB height and leakage $\mathrm{L}$ of $\mathrm{Np} 2$ and $\mathrm{Ns} 1$.

Fig.5. Characteristics of leakage L of insulated hybridtype transformer. 
It is found that our new type is more likely to increase leakage inductance because its primary wires board is made separately from the secondary ones. Factors affecting the leakage inductance of our new type are the length between primary wires board and secondary one as well as the thickness of primary wires board.

Magnetic field interpretation offers a clear explanation on the connection between the thickness of primary board and leakage inductance. Figure 6 shows the views of our new transformer and the comparative values of leakage inductance. Figures 6(a) and 6(b) are the wire structures of different models(A and $\mathrm{B}$ ) and results of magnetic field interpretation. Primary wires are installed on PCB using Np1-3 and TIW wires are covered with secondary ones. A thin primary PCB tends to decrease leakage inductance as shown in Fig. 6(c). The test results on $2.1 \mathrm{~mm}$ and $2.7 \mathrm{~mm}$ boards have produced a maximum of $7 \%$ differences from analysis. It appears that sample transformers produce a difference in leakage inductance, which seems to be caused by a difference in simulation models and the shape of second wires. This difference of $7 \%$ does not seem to have an effect on evaluating transformer properties.

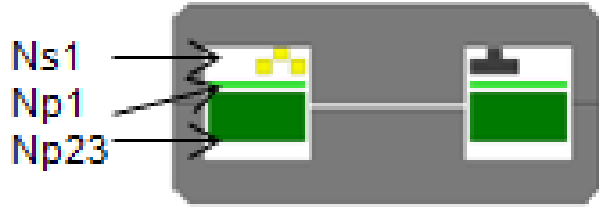

(a) Type-A wires structure.

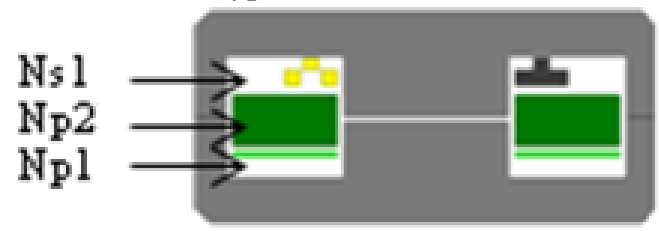

(b) Type-B wires structure.

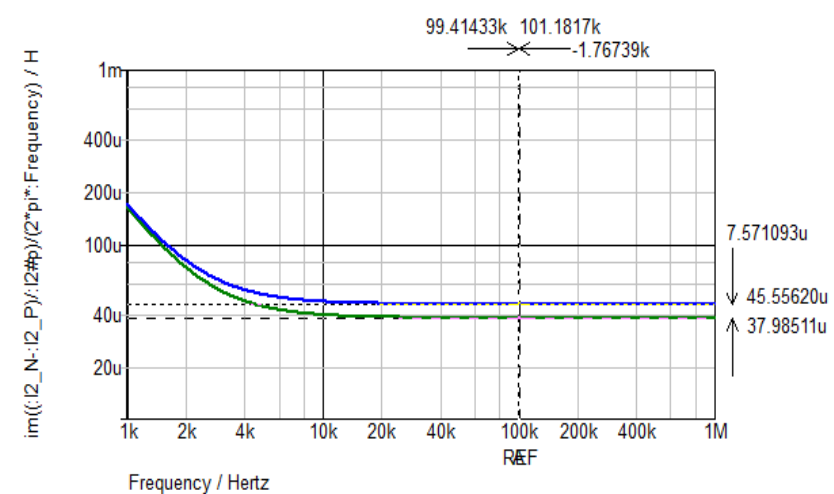

(c) Analysis results of leakage inductance of Type-A and Type-B

Fig.6. Characteristics of leakage inductance by wire type.

Our new transformer consists of three wires, two primary wires and one secondary wire. As for flyback converter, leakage inductance of primary main wires $(\mathrm{Np} 2)$ and secondary wires (Ns) have a connection with Vds voltage surge of main FET and secondary rectification diode surge. The characteristics of leakage inductance are considered to be strongly affected by the location of each wire when a PCB is made of secondary wires. Figure 6 shows characteristics of leakage inductance by wire location.
Leakage inductance of Type B has decreased by $7.6 \mathrm{uH}$ compared to that of Type-A. Such interpretation output has been applied to optimize the new transformer and generate leakage inductance up to the level of the conventional one.

B. EMI characteristics of AC adapters by transformer structure

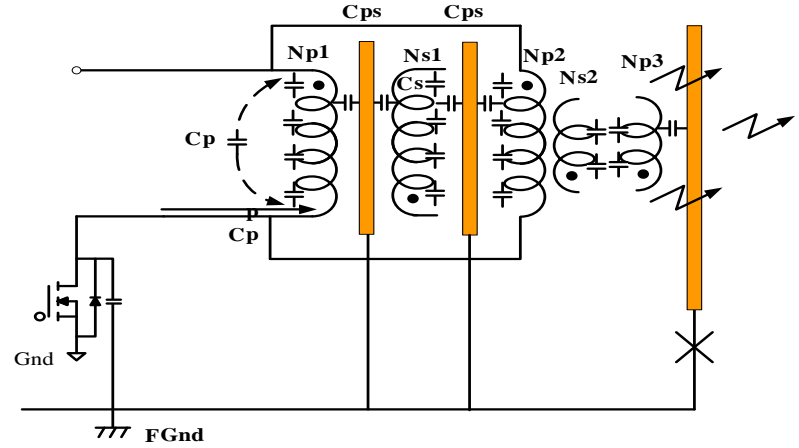

Fig.7. Internal noise path of transformer.

Since AC adapters should meet the requirement of EMI characteristics of CISPR22, FCC_ PART15 and EN550022, we have tested noise levels. Figure 6 shows internal noise path of transformer. Transformer has two kinds of parasitic capacitance which are $\mathrm{Cp}$, Cs Np1, Np2, Ns1 as well as Np1, Ns1, Cps. Parasitic capacitance makes a path to deliver noise to the primary side ${ }^{[6]}$. As figure 5(a) shows, copper shield is installed on PCB to decrease parasitic capacitance and increase impedance of noise path Figures 8 and 9 show the test results on EMI characteristics of AC adapters when the conventional and new type was installed. Conducted noise of the new type decreased by a maximum of $5 \mathrm{~dB}$ compared to the conventional-type. Radiated noise went down up to $15 \mathrm{~dB}$ in $70 \mathrm{MHz}$.

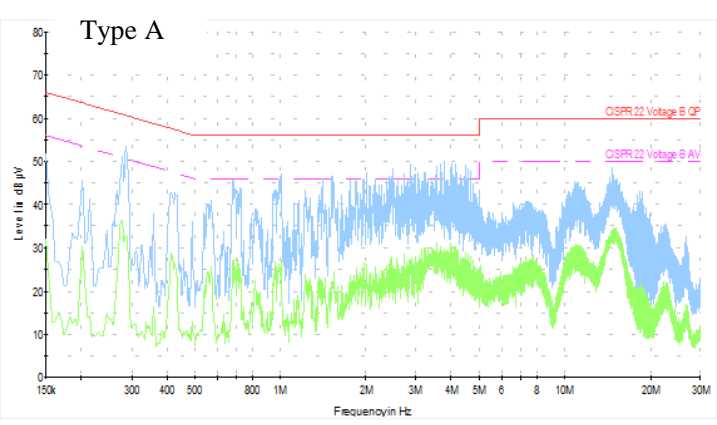

(a) Conducted noise spectrum

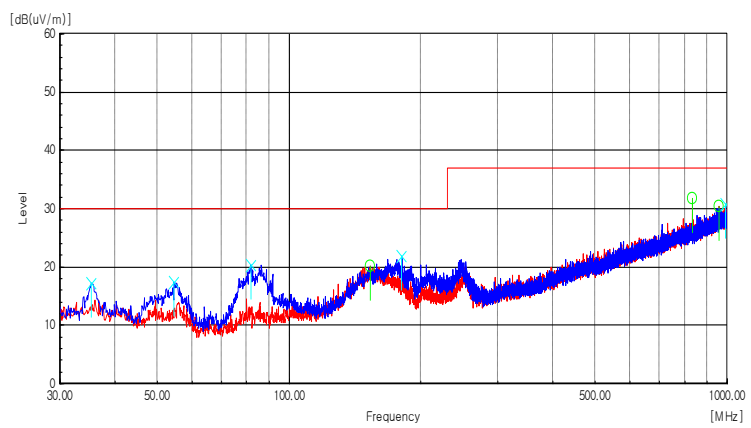

(b) Radiated Noise

Fig.8. EMI characteristics of AC adapters with the conventional-type transformer. 


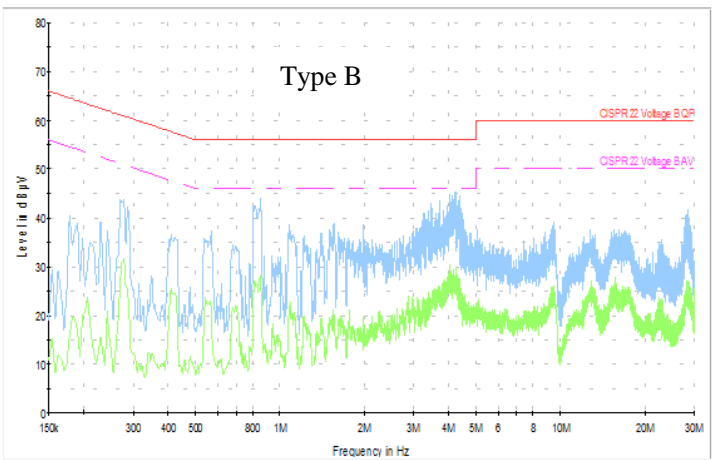

(a) Conducted noise spectrum.

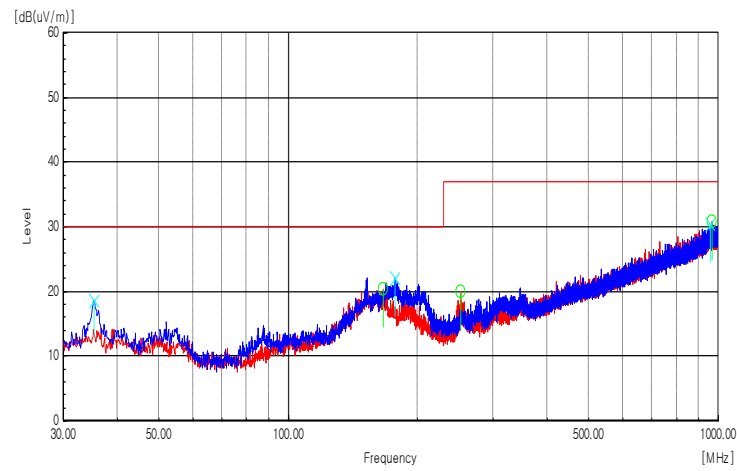

(b) Radiated Noise.

Fig.9. EMI characteristics of AC adapters with the original-type transformer.

C. Characteristics of power efficiency when mounted on AC adapters

Figure 10 shows the outside view of new and conventional type transformers. New type is approximately $45 \%$ smaller than the conventional one. The whole view of AC adaptor with new transformer is shown in the figure 10. The height of the new transformer mounted on the PCB decreased up to $7.0 \mathrm{~mm}$ compared to $10.95 \mathrm{~mm}$ of the conventional-type, which comes out to be reduced approximately by $25 \%$ of the entire height.

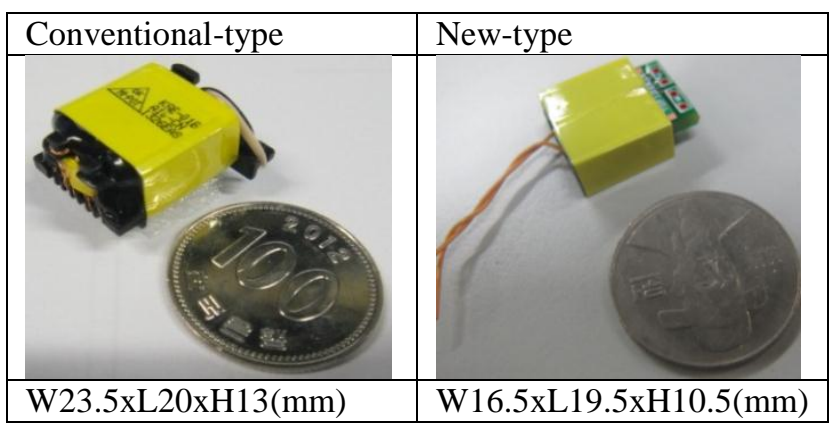

Fig. 10. The outside views and sizes of new and conventional transformers.

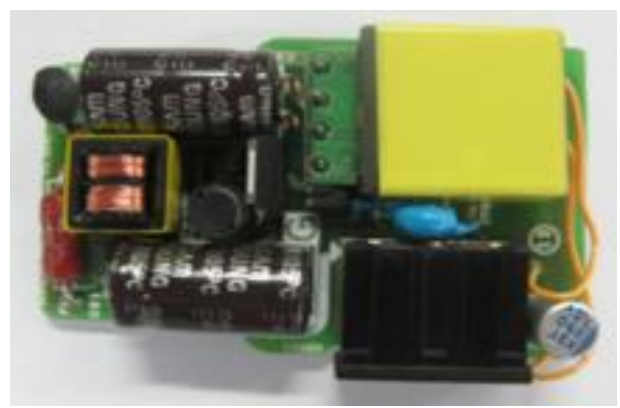

(a) Top view of AC adapter board.

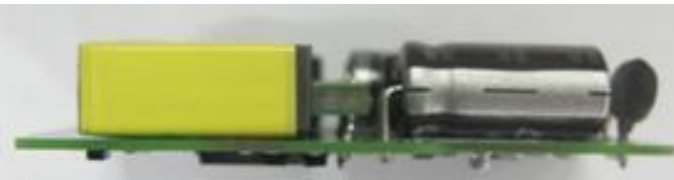

(b) Side view of AC adapter board.

Fig. 11. Outside view of the new transformer mounted on $\mathrm{AC}$ adapter.

Figure 12 shows efficiency characteristic of AC adapter power supply. The new-type is more effective than the conventional one by $2 \%$ at most.

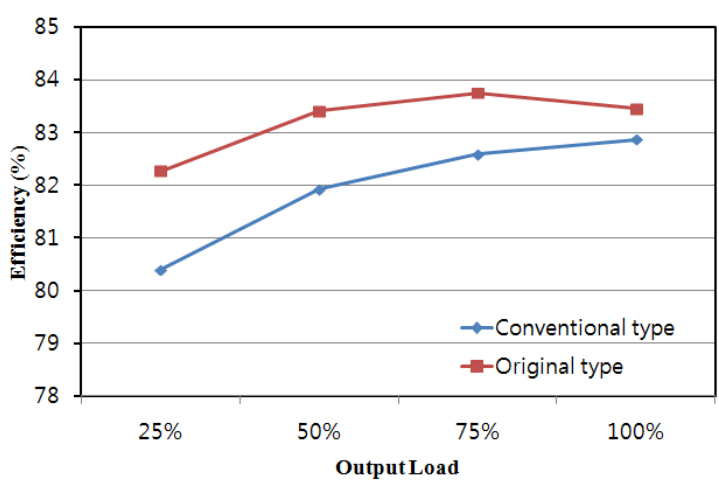

(a) Efficiency characteristics of input voltage AC115V.

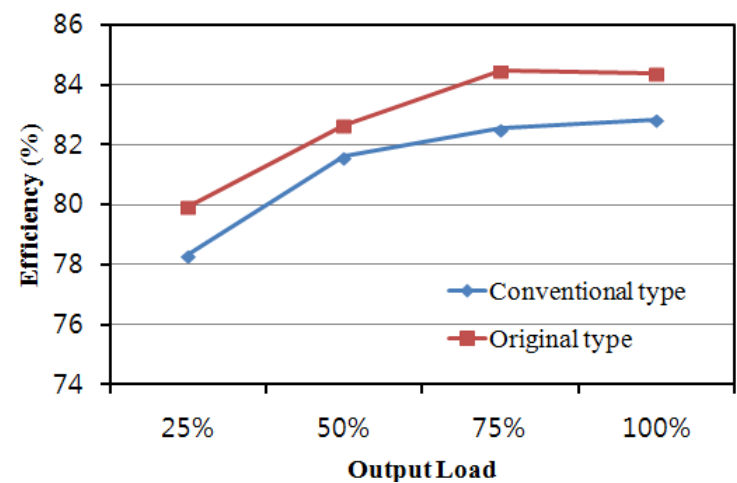

(b) Efficiency characteristics of input voltage AC230V Fig.12 Characteristics of power efficiency of AC adapter.

\section{CONCLUSION}

For the AC adaptors of smart devices, an ultra-small transformer has been developed by adopting an insulated hybrid structure with the size of W16.5 x L19.5 x H10.5 $\mathrm{mm}^{\wedge} 3$, whose volume is $65 \%$ smaller than conventional insulated transformers. Electrical features, structure and 
EMI characteristics of the new transformer have been he was with the Department of Nuclear Engineering, characterized by simulation and relevant tests. It also Hanyang University, Seoul, Korea.

compared power efficiency and EMI characteristics of each conventional and original transformer when they are mounted on AC adapters with the key findings as the following:

1. The size of the original-type transformer is approximately $65 \%$ smaller than the conventional one.

2. Conducted noise of the original-type transformer decreased up to $5 \mathrm{~dB}$ and radiated noise up to $15 \mathrm{~dB}$ compared to the conventional-type one.

3. Power efficiency of AC adapter went up by $2 \%$

4. The original-type transformer drove up production efficiency by $50 \%$.

Our new insulated hybrid-type transformer is expected to be further applied to not only $10 \mathrm{WAC}$ adapters but also other products.

\section{REFERENCES}

[1] M. Nymand, U. K. Madawala, M. A. E. Andersen, B. Carsten, O. S. Seiersen, "Reducing AC-Winding losses in high-current highpower inductors," in Proc, IEEE PESC 2009, pp.777-781.

[2] J. D. Pollock, C. R. Sulliven, "Modeling foil winding conFig.urations with low AC and DC resistance," in Proc. IEEE PESC 2005, pp.1507-1512

[3] C. Quinn, K. Rinne, T. O'Donnell, M. Duffy and C. O'Mathuna, "A review of planar magnetic techniques and technologies," in Proc. IEEE 2001, pp. 1175-1183.

[4] Anon., "New magnetic structure for a low profile planar transformer," Journal? vol.28, no. 2, July 1985, pp.625-626.

[5] N. Dai, A.W. LotFig.. Skutt, W Tabisz, F. C. Lee, "A comparative study of high frequency low profile planar transformer technologies", in Proc. APEC 1994, oo.153-161.

[6] G. Li, M. Shoyama, T. Ninomiya and I, Tokunari, "Common-mode noise reduction in DC-DC converters by common-dource type active-clamp technique," in Proc. IEEE PESC 2001. pp.1773-1178

[7] M.Albach, Th.Durbaum, A.Brockmer, "Calculating core losses in transformers for arbitrary magnetizing currents a comparison of different approaches," in Proc. IEEE PESC 1996. pp. 1463-1468

[8] Mulder, S.A, "Fit formulae for power loss in ferrites and their use in transformer design," in Proc. PCIM 1993. pp. 345-359

[9] Roshen,W., "Ferrite core loss for power magnetic components design, " 1991, IEEE Transaction in Magnetics 27, pp. 4407-4415

[10] R.prieto, J.A.Cobls, O.Garcia, R.Asensi and J.Uceda, "Optimizing the winding strategy of the transformer in a flyback conveter," in Proc. IEEE PESC 1996. pp. 1456-1462

\section{BIOGRAPHIES}

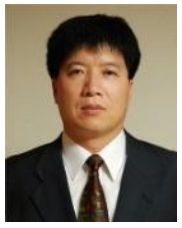

Heung Gyoon(Harry) Choi was born in Korea in 1965 .He received the B.S. degree in electronics in 1989 from Dankook University, Seoul, Korea, and He is currently working toward the M.S. and Ph.D. degree in Electrical Engineering from Hanyang University, Seoul, Korea. Since 1989, he has been with the Digital Module Division of Samsung Electro-Mechanics Co., Ltd., Suwon, Korea.

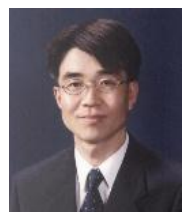

Kyu-Sun Chung received his B.S and M.S in Nuclear Engineering from Seoul National University, Seoul, Korea, in 1980 and 1982, respectively. His Ph.D of Applied Plasma Physics in Nuclear Engineering from Massachusetts Institute of Technology(M.I.T), USA, in 1989. From 1989 to 2005,
Since 2005, he has been an Professor with the Department of Engineering, Hanyang University.

His current research interests include plasma diagnostic, nuclear fusion and plasma processing.

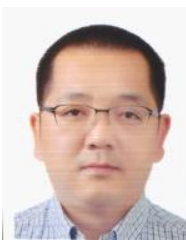

Ge Li received the B.S. degree in electronics in 1994 from Shenyang Technical University, Shenyang, China, and the M.E. and Dr. Eng. Degrees form Kyushu University, Fukuoka, Japan in 2001 and 2004 respectively.

He was engaged in the development of switching power supplies and their noise analysis from 2004 to 2009 at the power system business Grp, TDK Corporation, Chiba, Japan. He then worked on development for magnetic component for switching power supplies in the Digital module Division of Samsung Electro-Mechanics Co., Suwon, Korea.

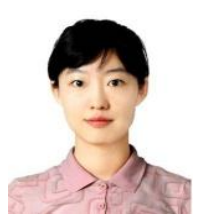

Xinlan Li, received her B.S from the University of Science and Technology Liaoning, Liaoning, China, in 2004, and her M.S and Ph. Dr from Gyeongsang National University, Gyeongnam, Korea in 2008 and 2012, respectively, where she was Post-doctor with research institute for Green Energy Convergence Technology in 2013. She has been with Department of Electrical Engineering, Catholic University of Korea, Deagu, where she was a Professor. Since 2014, She was currently with development for circuit technology for power supplies in the Digital module Division of Samsung Electro-Mechanics Co., Suwon, Korea. 